\title{
Structure of a B-DNA dodecamer: Conformation and dynamics*
}

\author{
(DNA structure/sugar puckering in DNA/principle of anticorrelation/torsion angles in DNA/thermal vibration in DNA)
}

\author{
Horace R. Drew ${ }^{\dagger}$, Richard M. Wing ${ }^{\dagger \ddagger}$, Tsunehiro Takano ${ }^{\dagger}$, Christopher Broka $^{\dagger \S}$, Shoji Tanaka ${ }^{\dagger 币}$, \\ KeIICHI Itakurall, AND RichaRd E. Dickerson ${ }^{\dagger}$
}

${ }^{\dagger}$ Norman W. Church Laboratory of Chemical Biology, California Institute of Technology, Pasadena, California 91125; and IICity of Hope National Medical Center, Duarte, California 91010

Communicated by P. D. Boyer, December 31, 1980

\begin{abstract}
The crystal structure of the synthetic DNA do-

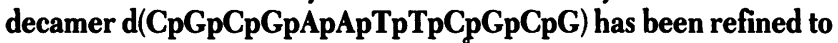
a residual error of $R=17.8 \%$ at $1.9-\AA$ resolution (two- $\sigma$ data). The molecule forms slightly more than one complete turn of righthanded double-stranded B helix. The two ends of the helix overlap and interlock minor grooves with neighboring molecules up and down a $2_{1}$ screw axis, producing a $19^{\circ}$ bend in helix axis over the 11-base-pair steps of the dodecamer. In the center of the molecule, where perturbation is least, the helix has a mean rotation of $36.9^{\circ}$ per step, or 9.8 base pairs per turn. The mean propeller twist (total dihedral angle between base planes) between $A \cdot T$ base pairs in the center of the molecule is $17.3^{\circ}$, and that between $C \cdot G$ pairs on the two ends averages $11.5^{\circ}$. Individual deoxyribose ring conformations as measured by the $\mathrm{C5}^{\prime}-\mathrm{C4}^{\prime}-\mathrm{C3}^{\prime}-\mathrm{O3}^{\prime}$ torsion angle $\delta$, exhibit an approximately Gaussian distribution centered around the $\mathrm{Cl}^{\prime}$ exo position with $\delta_{\text {avg }}=123^{\circ}$ and a range of $79^{\circ}$ to $157^{\circ}$. Purine sugars cluster at high $\delta$ values, and pyrimidine sugars cluster at lower $\delta$. A tendency toward 2-fold symmetry in sugar conformation about the center of the molecule is detectable in spite of the destruction of ideal 2-fold symmetry by the molecular bending. More strikingly, sugar conformations of paired bases appear to follow a "principle of anticorrelation," with $\delta$ values lying approximately the same distance to either side of the center value, $\delta=123^{\circ}$. This same anticorrelation is also observed in other DNA and DNA RNA structures.
\end{abstract}

In the 28 years since a double helix model for B-DNA was proposed by Watson and Crick (2), direct evidence for its structure has been based on refinement of models having standard bond parameters against $\mathrm{x}$-ray diffraction data from oriented fibers (3-6). This has had two disadvantages: loss of information because of rotational disorder about the fiber axis, and lack of information about the effect of specific base sequences, aside from a limited number of experiments on homopolymers and alternating copolymers. Recent advances in triester methods of DNA synthesis have made possible the preparation of molecules of predetermined base sequence, in quantities and purities suitable for single-crystal $x$-ray analysis. This paper presents the results of a structure analysis and refinement of a complete turn of right-handed B-DNA, with the sequence

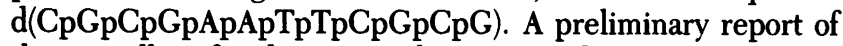
the partially refined structure has appeared (7).

Our structure analysis of the dodecamer C-G-C-G-A-A-T-TC-G-C-G is a logical extension of the earlier analyses of C-G-CG-C-G (8) and C-G-C-G (9). Both of the latter adopted a lefthanded zigzag or $\mathrm{Z}$ helix, a totally unexpected conformation whose relevance to biological DNA of more varied sequence became particularly interesting. In December 1979 we were fortunate in growing large single crystals of dodecamer, synthesized at Pasadena and Duarte. This sequence is of particular

The publication costs of this article were defrayed in part by page charge payment. This article must therefore be hereby marked "advertisement" in accordance with $18 \mathrm{U}$. S. C. $\$ 1734$ solely to indicate this fact. significance because it contains an EcoRI restriction site, G-AA-T-T-C, and because it brackets a Z-incompatible A-A-T-T segment with two Z-compatible C-G-C-G ends, offering a test of the tendency of mixed-sequence DNA to adopt the $\mathrm{Z}$ conformation. In spite of favorable salt conditions, the central A-A-T$\mathrm{T}$ segment apparently is sufficient to counteract the $\mathrm{Z}$-forming tendencies of the tetrameric C-G-C-G ends, resulting in a classical Watson-Crick B helix throughout.

Structure analysis and refinement

The dodecamer crystallizes in space group $\mathrm{P} 2{ }_{1} 2_{1} 2_{1}$ with $a=$ $24.87 \AA, b=40.39 \AA, c=66.20 \AA$, and two single strands or one double helix per asymmetric unit. Two isomorphous heavy atom derivatives were obtained by de novo triester synthesis with 5-bromodeoxycytidine at the third position along each strand and by diffusion of the anticancer agent cisplatin [cisdichlorodiamminoplatinum(II)] into pregrown crystals. Phases from this analysis were used to obtain a starting model for restrained least-squares refinement (10).

Initial energy parameters for DNA as obtained from Michael Levitt were modified so that conformational energy would restrain the molecule to a sterically acceptable structure but would allow the $x$-ray data to determine this structure. Internal sugar ring bond angles were set to the average of their $\mathrm{C} 2$ '-endo and $\mathrm{C}^{\prime}$-endo values but were left flexible enough that other conformations could be obtained (11). The residual error or $R$ factor for 2725 two- $\sigma$ data between 8.0 and $1.9 \AA$ was reduced from $42 \%$ to $18.1 \%$ by 50 cycles of position and temperature factor refinement. During this process, 10 superimposed Fourier/difference Fourier maps were inspected in order to make manual corrections and introduce solvent molecules. At the end of the 50 cycles, all 5534 zero- $\sigma$ data between 8.0 and $1.9 \AA$ were included in refinement. (Data out to $2.2-\AA$ resolution were measured at least twice on different crystals.) After 62 cycles, the final $R$ factor for 486 DNA atoms and 80 ordered solvent molecules is $23.9 \%$ for the complete zero- $\sigma$ data or $17.8 \%$ for the two- $\sigma$ data (calculated with the same parameter set). The worst bond length in the structure deviates by $0.03 \AA$ from its ideal value, and the worst bond angle deviates by $4.4^{\circ}$. Both intensity data and final coordinates have been deposited with the Brookhaven Protein Data Bank.

Nonuniform motion in the helix

The refined dodecamer structure is shown in Fig. 1. It is a righthanded, Watson-Crick B double helix with an average of 10.1 base pairs per turn over the entire helix. Two striking depar-

\footnotetext{
* This is paper no. 1 of a series; paper no. 2 is ref. 1.

₹ Present address: Department of Chemistry, University of California at Riverside, Riverside, CA 92521.

$\$$ Present address: Department of Chemistry, University of California at San Diego, La Jolla, CA 92093.

T Present address: Suntory Institute for Biomedical Research, Osaka, Japan.
} 

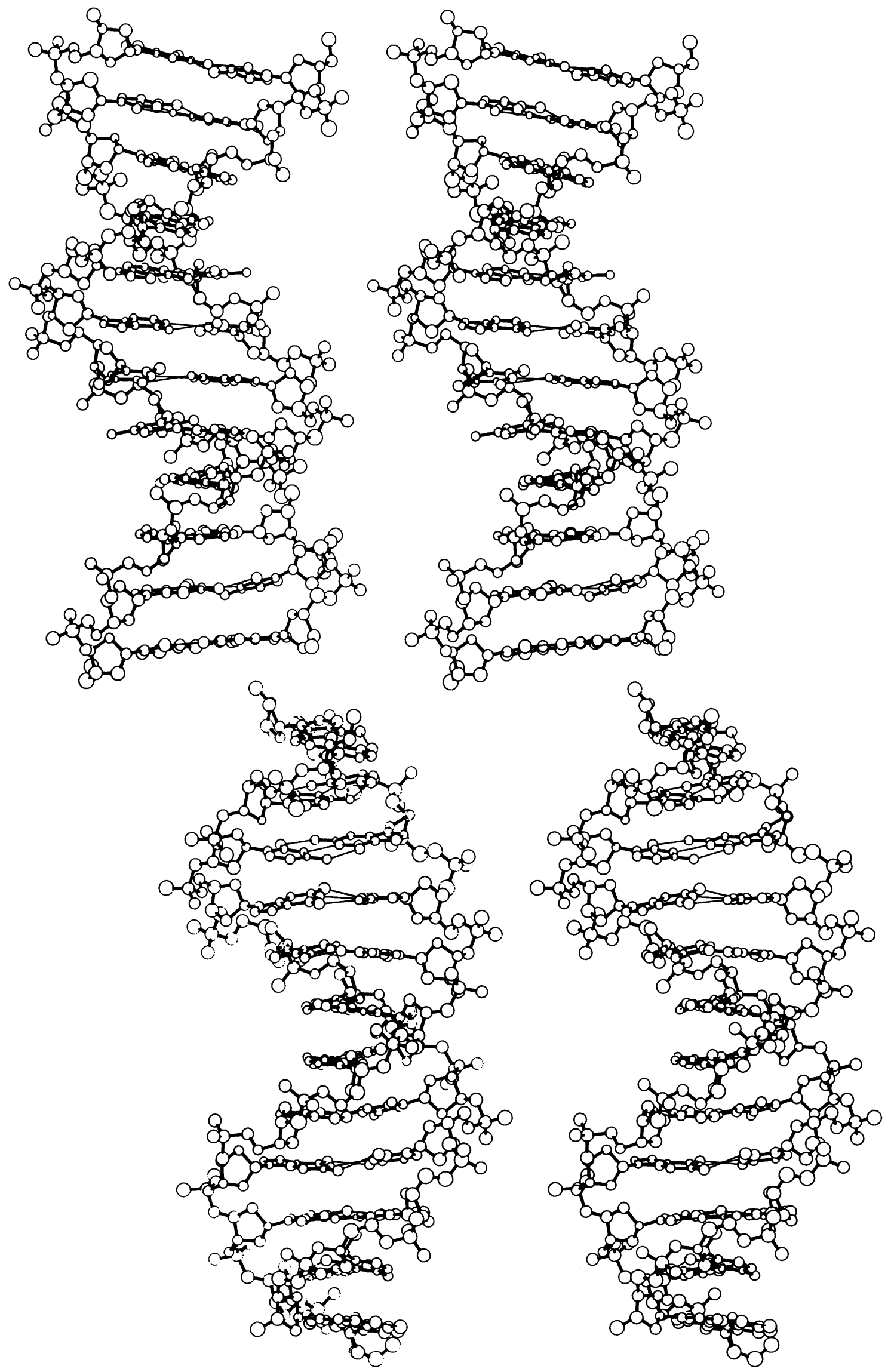

FiG. 1. "Vibration diagram" representation of the C-G-C-G-A-A-T-T-C-G-C-G double helix viewed into the wide groove (Upper) and $90^{\circ}$ to the right (Lower). Chains are identified in text and tables by consecutive base numbering $\mathrm{C1}, \ldots$. , G12 for one chain and $\mathrm{C13}, \ldots$. . G24 for the other. $\mathrm{C} 1$ is paired with G24, G2 with C23, . . , A5 with T20, etc. Base pair C1-G24 is at the top of each helix, and G12-C13 is at the bottom. The radius of each atom has been set to $u / 3 ; u$ is the rms displacement as obtained from its individual isotropic temperature factor parameter, $B=8 \pi^{2} u^{2}$. 
tures from the simplest classical B helix, discussed in the preliminary report (7), are the propeller twist of each individual base pair (total dihedral angle between base planes) and the $19^{\circ}$ bend in helix axis over 11 base pair steps. This bending is to the right in Fig. 1 Upper and concave toward the viewer in Fig. 1 Lower. Although probably induced by hydrogen bonds between molecules in the crystal, this bending requires less than $0.5 \mathrm{kcal} / \mathrm{mol}$ relative to a straight helix and illustrates the inherent flexibility of the DNA double helix.

The double helix structure is depicted in Fig. 1 in a manner that illustrates the relative displacement of atoms as calculated from individual isotropic temperature factors, $B$. The larger the atom, the greater is its mean displacement in the crystal structure, whether this is dynamic (thermal vibration) or static (positional variation between crystallographically equivalent molecules). Deoxyribose and phosphate group atoms on the outside of the helix are less restricted to a fixed position $\left(B_{\text {avg }}=42\right.$ and 51 , respectively) than are atoms within base pairs nearer the helix center $\left(B_{\text {avg }}=28\right)$. Part of this effect may arise from the inherent flexibility of a deoxyribose ring. Lacking a hydroxyl group on the 2 ' carbon atom, it does not experience the steric clash that a ribose ring does in shifting between $\mathrm{C} 3$ '-endo and C2'-endo conformations (11). For comparison purposes, in the only available example of a RNA polymer, tRNA (12), the temperature factors are more similar between phosphate groups $\left(B_{\text {avg }}=48\right)$ and base pairs $\left(B_{\text {avg }}=39\right)$.

The motion that we see in the crystal is entirely consistent with, but not necessarily identical to, the coupled sugarphosphate motion proposed to explain the nanosecond NMR relaxation times of DNA in solution (13). If the displacement seen in Fig. 1 is interpreted as molecular vibration, then the sugar-phosphate backbone on the outside vibrates with a greater amplitude than does the core of the helix, and base pairs at the upper and lower ends of the helix move more than those at the center. Atoms involved in the outer hydrogen bonds of any one base pair frequently vibrate more than those in the central hydrogen bond, consistent with a propeller twist motion. The reasonableness of the vibrational interpretation of the displacements in Fig. 1 is sufficient cause for favoring this explanation over the alternative one of static disorder within the crystal.

In many cases the deoxyribose ring appears to be rocking about the $\mathrm{Cl}^{\prime}-\mathrm{N}$ bond to the base, as suggested by the average $B$ values of 34 for $\mathrm{Cl}^{\prime}, 40$ for $\mathrm{C2}^{\prime}, 42$ for $\mathrm{C}^{\prime}$ and $\mathrm{Ol}^{\prime}$, and 44 for $\mathrm{C}^{\prime}$ '. Such a $\mathrm{Cl}^{\prime}-\mathrm{N}$ rocking motion could lead to a continuum of sugar conformations between $\mathrm{C}^{\prime}$ '-endo and $\mathrm{Ol}^{\prime}$-endo and, as will be seen in the following section, this is indeed what is observed in the dodecamer. However, a more detailed analysis (1) suggest that these represent genuine structural differences in conformation, rather than "frozen vibrations."

\section{Variations in sugar conformation}

Glycosyl and main-chain torsion angles for the refined dodecamer are listed in Table 1, along with ideal values that have been proposed for A- and B-DNA $(6,14)$. The main chain torsion angles $\alpha$ through $\zeta$ are close to the (gauche ${ }^{-}$, trans, gauche ${ }^{+}$, trans, trans, gauche ${ }^{-}$) values expected for an ideal B helix (5), with the greatest variation in the $\mathrm{C}^{\prime}-\mathrm{C}^{\prime}-\mathrm{C}^{\prime}-\mathrm{O} 3^{\prime}$ torsion angle $\delta$. The conformation of a deoxyribose ring is closely related to this angle in the manner shown along the top of Fig. 2 (15). For the dodecamer, the clarity of definition of the $\mathrm{C}^{\prime}-\mathrm{C} 4^{\prime}-\mathrm{C} 3^{\prime}-\mathrm{O} 3^{\prime}$ backbone chain in the electron density map permits the establishment of $\delta$ to within roughly $10^{\circ}$. The shape of each deoxyribose ring in the electron density map is compatible with the ring conformation predicted by torsion angle $\delta$, but the precision with which $\delta$ can be determined makes it the better guide to sugar conformation at less than atomic resolution (9).
Fig. 2 also illustrates the strong correlation between glycosyl angle $\chi$ and backbone torsion angle $\delta$. The observed conformations are scattered along a diagonal line in the plot, in a roughly Gaussian distribution about point $B$, which is one of the models for the B helix obtained by Levitt from energy considerations (14). This point corresponds to the $\mathrm{Cl}^{\prime}$-exo conformation, which was also encountered at each guanine position in left-handed Z-helical C-G-C-G (9). The C2'-endo conformation of a classical $B$ helix is represented by point $B_{F}$ at the upper right of the plot, and the $\mathrm{C}^{\prime}$ '-endo conformation of an A helix is at the far lower left, $A_{F}$, beyond the cluster of points from the dodecamer conformation.

One of the most striking aspects of Fig. 2 is the preference of purine sugars for high $\delta$ and $\chi$ values near the $C 2$ '-endo conformation and of pyrimidine sugars for low values nearer $\mathrm{Ol}^{\prime}$ endo, especially if the $\mathrm{Cl} \cdot \mathrm{G} 24$ base pair at the upper end of the helix is ignored as representing end effects. Examination of space-filling models suggests that a close contact exists between

Table 1. Main chain and glycosyl conformation angles

\begin{tabular}{|c|c|c|c|c|c|c|c|}
\hline \multirow{2}{*}{ Residue } & \multicolumn{7}{|c|}{ Angles, degrees } \\
\hline & $x$ & $\alpha$ & $\beta$ & $\gamma$ & $\delta$ & $\varepsilon$ & $\zeta$ \\
\hline C1 & -105 & - & - & 174 & 157 & -141 & -144 \\
\hline G2 & -111 & -66 & 170 & 40 & 128 & -186 & -98 \\
\hline C3 & -135 & -63 & 172 & 59 & 98 & -177 & -88 \\
\hline G4 & -93 & -63 & 180 & 57 & 156 & -155 & -153 \\
\hline A5 & -126 & -43 & 143 & 52 & 120 & -180 & -92 \\
\hline A6 & -122 & -73 & 180 & 66 & 121 & -186 & -89 \\
\hline $\mathrm{T} 7$ & -127 & -57 & 181 & 52 & 99 & -186 & -86 \\
\hline T8 & -126 & -59 & 173 & 64 & 109 & -189 & -89 \\
\hline C9 & -120 & -58 & 180 & 60 & 129 & -157 & -94 \\
\hline G10 & -90 & -67 & 169 & 47 & 143 & -103 & -210 \\
\hline C11 & -125 & -74 & 139 & 56 & 136 & -162 & -90 \\
\hline G12 & -112 & -82 & 176 & 57 & 111 & - & - \\
\hline C13 & -128 & - & - & 56 & 137 & -159 & -125 \\
\hline G14 & -116 & -51 & 164 & 49 & 122 & -182 & -93 \\
\hline C15 & -134 & -63 & 169 & 60 & 86 & -185 & -86 \\
\hline G16 & -115 & -69 & 171 & 73 & 136 & -186 & -98 \\
\hline A17 & -106 & -57 & 190 & 54 & 147 & -183 & -97 \\
\hline A18 & -108 & -57 & 186 & 48 & 130 & -186 & -101 \\
\hline T19 & -131 & -58 & 174 & 60 & 109 & -181 & -88 \\
\hline $\mathrm{T} 20$ & -120 & -59 & 179 & 55 & 122 & -181 & -94 \\
\hline C21 & -114 & -59 & 185 & 45 & 110 & -177 & -86 \\
\hline G22 & -88 & -67 & 179 & 50 & 150 & -100 & -188 \\
\hline C23 & -125 & -72 & 139 & 45 & 113 & -174 & -97 \\
\hline G24 & -135 & -65 & 171 & 47 & 79 & - & - \\
\hline Mean & -117 & -63 & 171 & $54^{*}$ & 123 & -169 & -108 \\
\hline$\pm \mathrm{SD}$ & 14 & 8 & 14 & 8 & 21 & 25 & 34 \\
\hline $\mathrm{B} \mathrm{DNA}^{\dagger}$ & -119 & -61 & 180 & 57 & 122 & -187 & -91 \\
\hline $\mathrm{B}_{\mathrm{F}}$ DNA $\ddagger$ & -102 & -41 & 136 & 38 & 139 & -133 & -157 \\
\hline $\mathrm{A}_{\mathrm{F}} \mathrm{DNA}^{\S}$ & -154 & -90 & -149 & 47 & 83 & -175 & -45 \\
\hline
\end{tabular}

Main chain conformation angles are defined as:

$$
{ }^{\alpha}{ }^{\beta}-05^{\prime}-\mathrm{C} 5^{\prime}-\mathrm{C} 4^{\prime}-\mathrm{C} 3^{\prime}-03^{\prime}-\mathrm{P}
$$

with 0 at the fully eclipsed or cis position and positive clockwise rotation of the farther pair of atoms. The glycosyl angle $\chi$ is similarly defined in terms of atoms $01^{\prime}-\mathrm{C} 1^{\prime}-\mathrm{N} 1-\mathrm{C} 2$ for pyrimidines $(\mathrm{C}$ and $\mathrm{T})$ or $01^{\prime}-\mathrm{C} 1^{\prime}-\mathrm{N} 9-\mathrm{C} 4$ for purines (G and $\left.\mathrm{A}\right)$.

* Omitting $\mathrm{C} 1$ value.

† Model chosen from Levitt (14) with energy refinement for best agreement with our results: 10 base pairs per turn and $\mathrm{C}-\mathrm{O}-\mathrm{C}$ deoxyribose angle set at $115^{\circ}$.

₹ Fiber data of Arnott et al. (6) with 10 base pairs per turn.

$\S$ Fiber data of Arnott et al. (6) with 11 base pairs per turn. 


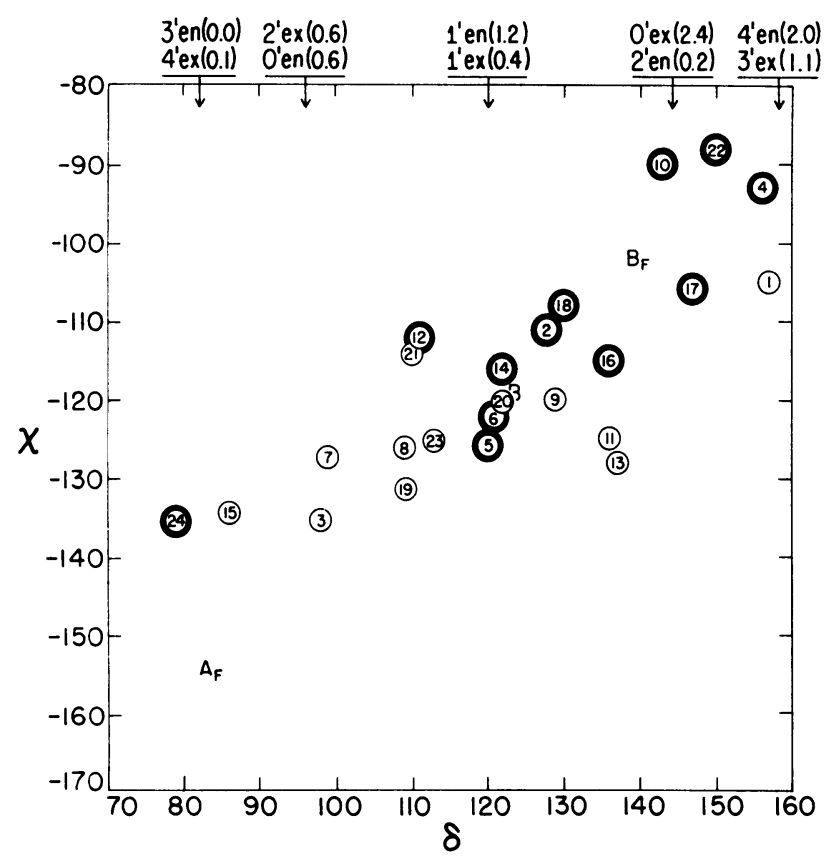

FIG. 2. Correlation plot between glycosyl torsion angle $\chi$ and the $\mathrm{C5}^{\prime}-\mathrm{C4}^{\prime}-\mathrm{C4}^{\prime}-\mathrm{O} 3^{\prime}$ torsion angle $\delta$ (or $\psi^{\prime}$ ). Each sugar is represented by a numbered circle, with angles taken from Table $1 . A_{F}, B_{F}$, and $B$ locate ideal $A$ and $B$ helices as derived from fiber diffraction (6) or energy refinement (14). The distribution of conformations is close to Gaussian centered at point $B$. Purines are shown by heavy circles, and pyrimidines, by light ones. Notice the correlation (similar $\delta$ values) between bases related by a 2 -fold axis normal to the helix (1/13, 2/14, etc.), and the anticorrelation (constant $\delta$ sum) between bases that are paired together on one step of the helix $(1 / 24,2 / 23$, etc.). The $\delta$ values for the 10 standard endo and exo conformations and their energies in kcal/ mol relative to $\mathrm{C3}^{\prime}$-endo are given along the top of the plot (15).

the $\mathrm{H}$ atom of a sugar $\mathrm{Cl}^{\prime}$ and the $\mathrm{O} 2$ of a pyridimine at $\chi=$ $-120^{\circ}$. This contact cannot be relieved by making $\chi$ less negative without introducing other clashes between the pyrimidine $\mathrm{H} 6$ and the hydrogens attached to either $\mathrm{C} 2{ }^{\prime}$ or $\mathrm{C}^{\prime}$, but a shift to more negative $\chi$ eliminates the clash. In contrast, the larger angle involved in the connection of a sugar to the five-membered ring of a purine keeps the N3 and $\mathrm{H} 8$ atoms far enough away from the sugar ring that no close contacts result. Hence, the purine sugars are free to adopt higher $\chi$ values and an ideal $\mathrm{C} 2$ '-endo conformation.

In view of the gentle slope of the total energy curve in the vicinity of point B (figure 2 of ref. 15), the scatter in individual conformations seen in Fig. 2 is hardly surprising. The "ideal" B helix in DNA is as much an oversimplification as is the "ideal" $\alpha$ helix in proteins. The DNA is not aware of the discrete conformational states listed at the top of Fig. 2, only of a smooth and rather shallow potential well centered in the vicinity of point $\mathrm{B}$. Visual inspection of the stereo drawings indicates that conformations can best be described as $\mathrm{C}^{\prime}$ '-endo for all sugars to the right of $\delta=129^{\circ}$ in Fig. 2, $\mathrm{Cl}^{\prime}$-exo for sugars between $105^{\circ}$ and $129^{\circ}$, and $01^{\prime}$-endo for sugars 15,3 , and 7 , with one lone $\mathrm{C} 3^{\prime}$-endo or A-like sugar puckering at the $3^{\prime}$ terminus of the second strand. (This assignment is necessarily arbitrary and subjective at intermediate angles.)

Two other features of the distribution in Fig. 2 deserve special mention. Sugars related by an approximate 2 -fold axis through the center of the molecule perpendicular to the helix axis ( 1 and 13, 2 and 14, 3 and 15, etc.) tend to have similar $\delta$ and $\chi$ values, indicating local preservation of 2 -fold symmetry in spite of the overall $19^{\circ}$ bend in the helix axis visible in Fig. 1 Upper (and discussed in ref. 7). Such 2-fold-related sugars differ in $\delta$ by a mean $\left( \pm\right.$ SD) of only $16.5^{\circ} \pm 8.3^{\circ}$. This is to be expected, and the only unusual feature is that this conformational symmetry is preserved in spite of the bending of the helix axis.

The second correlation is more surprising and potentially of more fundamental significance. The conformations of sugars in paired bases $(1 / 24,2 / 23,3 / 22$, etc. $)$ tend to be anticorrelated: if one conformation lies to the left of center in Fig. 2, the other conformation lies a similar distance to the right of center. This is a stronger statement than the previous observation that purines generally prefer higher $\delta$ and $\chi$ values than do pyrimidines. The midpoint in $\delta$ for each base pair have a mean of $122.8^{\circ}$, near the $\mathrm{Cl}^{\prime}$-exo conformation, with a mean deviation of only $5.6^{\circ}$. Some base pairs such as $5 / 20,6 / 19$, and $2 / 23$ have similar sugar conformations centered closely at $\mathrm{Cl}^{\prime}$-exo. Others such as $1 / 24,10 / 15$, and $3 / 22$ have quite disparate conformations, one of them nearer $\mathrm{C} 2$ '-endo or $\mathrm{C} 3$ '-exo and the other near $\mathrm{Ol}^{\prime}$-endo or even $\mathrm{C}^{\prime}$ '-endo. If the difference between $\delta$ values for two paired bases is defined as the conformational spread, then the $C \cdot G$ base pairs have a larger mean spread $\left(36.9^{\circ}\right)$ than do the $\mathrm{A} \cdot \mathrm{T}$ base pairs $\left(20.8^{\circ}\right)$, although the statistical significance of this is not clear with only 12 base pairs to compare.

This behavior of sugar conformations in paired bases is sufficiently striking to be formalized by defining it as the "principle of anticorrelation": deoxyribose sugars attached to paired bases in B-DNA tend to adopt $\delta$ values that are equidistant to either side of a central $\delta=123^{\circ}$ (or $\mathrm{Cl}^{\prime}$-exo) value. It probably is a consequence of wrapping sugar-phosphate chains having flexible deoxyribose rings around base pairs of fixed dimensions. One would not necessarily expect to find anticorrelation in sugar conformations of paired bases in RNA, with its more constrained ribose ring, and, indeed, such anticorrelation is not present in the one available example, tRNA (16). When the midpoints in $\delta$ values for paired bases in tRNA are calculated, omitting the first base pair at either end of a double-helical stack to eliminate end effects, the mean of these values is $82.8^{\circ}$ and the mean deviation is $2.4^{\circ}$. But the mean spread or difference in $\delta$ values between paired bases is only twice this, $5.4^{\circ}$. The half-spread from the midpoint is no larger than the uncertainty in midpoint position, indicating no statistically significant anticorrelation in sugar conformation. By comparison, in the DNA dodecamer CG-C-G-A-A-T-T-C-G-C-G the mean is $122.8^{\circ}$, the mean deviation is $5.6^{\circ}$, and the mean spread over all base pairs is $31.5^{\circ}$, 6 times as great.

The anticorrelation principle also is observed in the nonintercalated $\mathrm{T} \cdot \mathrm{A}$ base pair step of the $2: 1$ intercalation complex of daunomycin with C-G-T-A-C-G (17). Sugar pucker information provided in ref. 17 indicates that the Cs and Gs bracketing the intercalator molecules would be scattered at the upper right of Fig. 2 with the exception of an anomalous $\mathrm{Cl}$ conformation at the beginning of the chain. But the unintercalated T3.A4 is a beautiful example of anticorrelation. (Because the complex has an internal 2-fold axis of crystallographic symmetry, the two strands are identical. T3 on one strand is followed by $\mathrm{A} 4$ on the same strand and hydrogen bonded to $\mathrm{A} 4$ on the opposite strand.) T3 has the $(\chi, \delta)$ conformation $\left(-131^{\circ}, \approx 96^{\circ}\right)$, and $\mathrm{A} 4$ has $\left(-107^{\circ}, \approx 144^{\circ}\right)$. (The $\chi$ values quoted in ref. 17 should have $180^{\circ}$ subtracted from each to bring them into accord with IUPAC-IUB conventions.) In addition, the tendency, noted by both Sobell and colleagues (18) and Rich et al . $(19,20)$, for many intercalating groups (but not daunomycin) to induce a (C3'-endo) -3', 5'-(C2'-endo) conformation at the bases bracketing the intercalator, whether in DNA or RNA helices, can be regarded as an extreme limit of the principle of anticorrelation. Zimmerman and Pheiffer (21) have recently found a RNA $\cdot$ DNA hybrid, poly $(\mathrm{rA}) \cdot \operatorname{poly}(\mathrm{dT})$, that can be induced to adopt a B helix under conditions of high humidity. Its $\delta$ values as deduced from 
Table 2. Local helix parameters

\begin{tabular}{lrlrl}
\hline $\begin{array}{l}\text { Base } \\
\text { pairs }\end{array}$ & $\begin{array}{r}\text { Propeller } \\
\text { twist, }{ }^{\circ}(\psi)\end{array}$ & $\begin{array}{c}\text { Helix twist } \\
\text { angle, }{ }^{*}(\theta)\end{array}$ & $\begin{array}{c}\text { Base pairs per } \\
\text { turn }^{\dagger}(n)\end{array}$ & $\begin{array}{c}\text { Rise per base } \\
\text { pair, } \AA(h)\end{array}$ \\
\hline C1/G24 & $13.2 \pm 2.0$ & $38.3 \pm 1.1$ & $9.40 \pm 0.27$ & $3.36 \pm 0.01$ \\
G2/C23 & $11.7 \pm 2.1$ & $39.6 \pm 6.1$ & $9.09 \pm 1.40$ & $3.38 \pm 0.08$ \\
C3/G22 & $7.2 \pm 2.1$ & $33.5 \pm 2.1$ & $10.75 \pm 0.67$ & $3.26 \pm 0.05$ \\
G4/C21 & $13.2 \pm 1.9$ & $37.4 \pm 1.7$ & $9.63 \pm 0.44$ & $3.30 \pm 0.10$ \\
A5/T20 & $17.1 \pm 2.1$ & $37.5 \pm 0.9$ & $9.60 \pm 0.23$ & $3.27 \pm 0.02$ \\
A6/T19 & $17.8 \pm 2.1$ & $32.2 \pm 2.1$ & $11.18 \pm 0.73$ & $3.31 \pm 0.03$ \\
T7/A18 & $17.1 \pm 1.9$ & $36.0 \pm 2.8$ & $10.00 \pm 0.78$ & $3.29 \pm 0.01$ \\
T8/A17 & $17.1 \pm 2.0$ & $41.4 \pm 2.1$ & $8.70 \pm 0.42$ & $3.14 \pm 0.02$ \\
C9/G16 & $18.6 \pm 1.9$ & $32.3 \pm 1.3$ & $11.11 \pm 0.45$ & $3.56 \pm 0.07$ \\
G10/C15 & $4.9 \pm 1.9$ & $44.7 \pm 5.4$ & $8.05 \pm 0.97$ & $3.21 \pm 0.18$ \\
C11/G14 & $17.2 \pm 1.9$ & $37.0 \pm 1.9$ & $9.73 \pm 0.50$ & $3.54 \pm 0.19$ \\
G12/C13 & $6.2 \pm 2.3$ & & & \\
& & & & \\
Mean & $13.4 \pm 4.9$ & $37.3 \pm 3.8$ & $9.75 \pm 0.98$ & $3.33 \pm 0.13$ \\
& & & & \\
A DNA & & & 11.0 & 2.56 \\
(5, 22) & & 32.7 & & \\
B DNA & & & & \\
(5, 22, 23) & & 36.0 & 10.0 & 3.38 \\
C DNA & & & & \\
(23) & & 38.6 & 9.33 & 3.31 \\
D DNA & & 45.0 & 8.0 & 3.03 \\
(23) & & & &
\end{tabular}

Helical parameters were found by using vectors between atoms $\mathrm{C1}^{\prime}$ and the attached $\mathrm{N}$ of one base and the equivalent atoms of the next base up the same chain, with a program kindly provided by John Rosenberg. Standard deviations are shown at individual steps and statistical variation over the entire helix is shown with the means. Propeller twist is the dihedral angle between base planes in the same base pair and is twice the fiber twist in 22 and 23.

${ }^{*}$ Rotation per base pair.

$+n=360 / \theta$.

fiber diffraction patterns are $97^{\circ}$ and $152^{\circ}$ for rA and dT, respectively. The midpoint of these values, $124.5^{\circ}$, is only $1.7^{\circ}$ away from the mean DNA dodecamer value.

\section{Local helix parameters}

A helix-generating program was used to determine the rotation angle, orientation of the local helix rotation vector, and rise per base pair along the local axis for each of the 11 base pair steps along the dodecamer. The individual rotation angles and rise per base pair are listed in Table 2, along with the measured propeller twist at each base pair. These numbers probably are more accurate than any other results of the analysis, because base planes are well defined in the map and are established by a large number of atomic positions. The propeller twist of $A \cdot T$ base pairs in the center of the molecule is a remarkably uniform $17.3^{\circ} \pm 0.4^{\circ}$ (mean $\pm \mathrm{SD}$ ), and much of the variation at the two ends probably arises from overlapping of helices up the $c$ axis (figure 3 of ref. 7). The smaller mean, $11.5^{\circ} \pm 5.1^{\circ}$ for the two C-G-C-G ends, could also reflect the flattening influence of the third hydrogen bond in each base pair.

The individual helical twist or rotation values for the five central base pair steps that are unperturbed by overlapping ends (from G4 to C9) give a mean of $36.9^{\circ} \pm 3.3^{\circ}$, corresponding to 9.8 base pairs per turn. The three base pair steps at either end are wound somewhat more tightly, $37.6^{\circ} \pm 4.5^{\circ}$ or 9.6 base pairs per turn. The overall value of $35.8^{\circ}$ or 10.1 base pairs per turn that was reported in ref. 7 arises because several of the local twist vectors in the overlapping ends of the molecule are sharply tilted from the mean helix axis, altering their twist angles in projection. Space does not permit a detailed discussion of local and global helix twist vectors here, but this is discussed in detail elsewhere (1). It can only be mentioned here that these displacements in local helix rotation vectors show a 2 -fold symmetry about an axis through the center of the molecule, perpendicular to the page in Fig. 1 Upper or horizontal in Fig. 1 Lower. This ideal symmetry axis is expected from the chemical identity of the two strands but is destroyed by the $19^{\circ}$ bend in the helix axis. Hence, these local variations in helix twist vector are observed in spite of the bend in the helix, not because of it.

We thank Peter Dembek for his help in synthesis of the dodecamer and Lillian Casler for her assistance in preparing the figures. This work was carried out with the support of National Institutes of Health Grants GM-12121 and GM-24393 and National Science Foundation Grant PCM79-13959. H.R.D. was the recipient of a National Institutes of Health Predoctoral Traineeship. This is contribution no. 6317 from the Division of Chemistry and Chemical Engineering.

1. Dickerson, R. E. \& Drew, H. R. (1981) J. Mol. Biol., in press.

2. Watson, J. D. \& Crick, F. H. C. (1953) Nature (London) 171 , 737-738.

3. Langridge, R., Wilson, H. R., Hooper, C. W., Wilkins, M. H. F. \& Hamilton, L. D. (1960) J. Mol. Biol. 2, 19-37.

4. Fuller, W., Wilkins, M. H. F., Wilson, H. R., Hamilton, L. D. \& Arnott, S. (1965) J. Mol. Biol. 12, 60-80.

5. Arnott, S. \& Hukins, D. W. L. (1972) Biochem. Biophys. Res Commun. 47, 1504-1509.

6. Arnott, S., Chandrasekaran, R., Birdsall, D. L., Leslie, A. G. W. \& Ratliff, R. L. (1980) Nature (London) 283, 743-745.

7. Wing, R. M., Drew, H. R., Takano, T., Broka, C., Tanaka, S., Itakura, K. \& Dickerson, R. E. (1980) Nature (London) 287, 755-758.

8. Wang, A. H.-J., Quigley, G. J., Kolpak, F. J., Crawford, J. L., Van Boom, J. H., Van der Marel, G. \& Rich, A. (1979) Nature (London) 282, 680-686.

9. Drew, H. R., Takano, T., Tanaka, S., Itakura, K. \& Dickerson, R. E. (1980) Nature (London) 286, 567-573.

10. Jack, A. \& Levitt, M. (1978) Acta Crystallogr. A34, 931-935.

11. Westhof, E. \& Sundaralingam, M. (1980) J.Am. Chem. Soc. 102, 1493-1500.

12. Sussman, J. L., Holbrook, S. R., Warrant, R. W., Church, G. M. \& Kim, S.-H. (1978) J. Mol. Biol. 123, 607-630.

13. Hogan, M. E. \& Jardetzky, O. (1980) Biochemistry 19, 3460-3468.

14. Levitt, M. (1978) Proc. Natl. Acad. Sci. USA 75, 640-644.

15. Levitt, M. \& Warshel, A. (1978) J. Am. Chem. Soc. 100, 2607-2613.

16. Holbrook, S. R., Sussman, J. L., Warrant, R. W. \& Kim, S.-H. (1978) J. Mol. Biol. 123, 631-660.

17. Quigley, G. J., Wang, A. H.-J., Ughetto, G., van der Marel, G., van Boom, J. H. \& Rich, A. (1980) Proc. Natl. Acad. Sci. USA 77, 7204-7208.

18. Jain, S. C., Tsai, C.-C. \& Sobell, H. M. (1977) J. Mol. Biol. 114, $317-331$.

19. Rich, A., Quigley, G. J. \& Wang, A. H.-J. (1979) in Stereodynamics of Molecular Systems, ed. Sarma, R. H. (Pergamon, New York), pp. $315-330$.

20. Rich, A., Wang, H.-J. \& Quigley, G. J. (1980) in Frontiers of Bioorganic Chemistry and Molecular Biology, ed. Ananchenko, S. N. (Pergamon, New York), pp. 327-337.

21. Zimmerman, S. B. \& Pheiffer, B. H. (1980) Proc. Natl. Acad. Sci. USA 78, 78-82.

22. Arnott, J., Dover, S. D. \& Wonacott, A. J. (1969) Acta Crystal$\log r$. B25, 2192-2206.

23. Arnott, J. \& Selsing, E. (1975) J. Mol. Biol. 98, 265-269. 